\title{
Actividad antifúngica de bacterias endófitas para el control de Fusarium
}

\author{
verticillioides en maíz
}

\section{Antifungal activity of endophytic bacteria controlling Fusarium verticillioides in}

\author{
maize
}

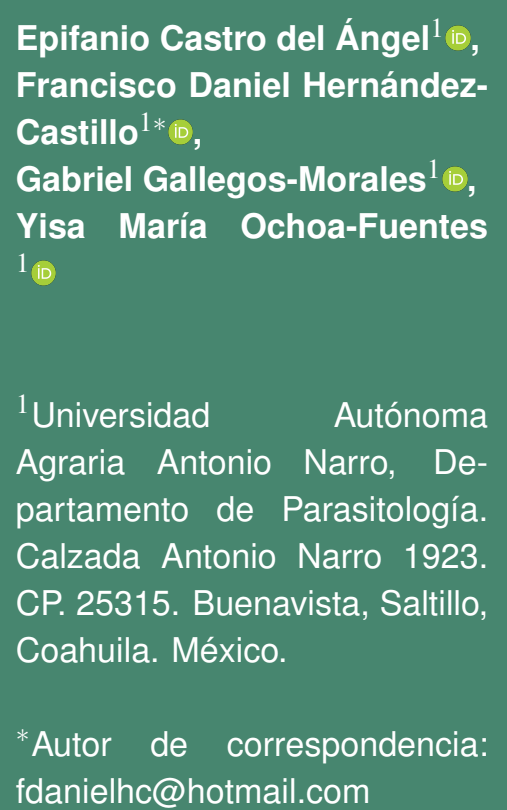

Nota científica

Recibida: 08 de enero 2021

Aceptada: 03 de agosto 2021

Como citar: Castro del Ángel E, Hernández-Castillo FD, Gallegos-Morales

$\mathrm{G}$, Ochoa-Fuentes YM (2021) Actividad antifúngica de bacterias endófitas para el control de Fusarium verticillioides en maíz . Ecosistemas y Recursos Agropecuarios 8(2): e2790. DOI: 10.19136/era.a8n2.2790
RESUMEN. El objetivo de esta investigación fue conocer la actividad antifúngica de dos aislamientos endófitos de Bacillus amyloliquefaciens contra Fusarium verticillioides bajo condiciones de laboratorio e invernadero. La inhibición in vitro en cultivos duales del hongo fitopatógeno se obtuvo en un rango del 59 al $62 \%$. Las bacterias endófitas redujeron la incidencia y la severidad de $F$. verticillioides más del $80 \%$ en las plantas que recibieron el tratamiento con respecto al testigo. La aplicación individual de las bacterias endófitas incrementó el contenido de clorofila en las hojas más del 150\% que el testigo. Los niveles de clorofila, el diámetro de tallo y el peso del fruto incrementó en las plantas que recibieron el tratamiento de las cepas antagonistas. La corroboración molecular de las cepas bacterianas indicó el 99\% de identidad con Bacillus amyloliquefaciens contenidas en el banco de datos del GenBank con clave de acceso KU570451.1 y KX665550.1.

Palabras clave: Biocontrol, Bacillus amyloliquefaciens, incidencia, promoción de crecimiento, severidad.

ABSTRACT. The objective of this research was to know the antifungal activity of two endophytic isolates of Bacillus amyloliquefaciens against Fusarium verticillioides under laboratory and greenhouse conditions. In vitro dual cultures inhibition of phytopathogenic fungus was detected in a range of 59 to $62 \%$. The endophytic bacteria reduced $F$. verticillioides incidence and severity more than $80 \%$ in plants that received the treatment compared to the control. The individual application of endophytic bacteria increased the chlorophyll content in leaves more than $150 \%$ compared to the control. Chlorophyll levels, stem diameter and fruit weight increased in all plants that received the antagonist strains treatment. The molecular corroboration of bacterial strains indicated $99 \%$ of identity withBacillus amyloliquefaciens contained in GenBank database with access codes KU570451.1 and KX665550.1.

Key words: Biocontrol, Bacillus amyloliquefaciens, growth promotion, incidence, severity. 


\section{INTRODUCCIÓN}

La pudrición del tallo, la mazorca y la raíz del maíz causada por Fusarium verticillioides afecta la producción de cultivos en las regiones tropicales y subtropicales (Figueroa-López et al. 2016, Kuki et al. 2020); se transmite por el suelo y es responsable de las principales pérdidas de rendimiento además de causar pérdidas de calidad debido a la acumulación de micotoxinas en los granos (Einloft et al. 2021). Las micotoxinas son un grupo de metabolitos secundarios producidas por hongos filamentosos, las cuales causan daños a la salud humana y animal (Caglayan et al. 2020).

Una de las toxinas mayormente producidas por F. verticillioides son las fumonisinas (Kamle et al. 2019), que inducen toxicidad pleiotrópica en animales, incluyendo neurotoxicidad, nefrotoxicidad y hapatotoxicidad con un impacto relevante en la salud humana (Liu et al. 2019). En humanos están asociadas con apoptosis, cáncer de esófago y defectos del tubo neural (Ahangarkani et al. 2014). La falta y escasa accesibilidad de métodos de control eficaces y ambientalmente seguros han llevado a un mayor interés en alternativas prácticas y biológicas para reducir la ingesta de fumonisinas; los métodos químicos y agronómicos que previenen la pudrición de la mazorca de maíz no son eficientes debido a que F. verticillioides infecta sistemicamente sin producir síntomas, pudiéndose propagar desde la semilla al grano (Wang et al. 2016).

El control biológico es una alternativa prometedora y ecológicamente amigable en el manejo de enfermedades de las plantas, se reportan varios agentes de control biológico como posibles biofungicidas (Nasir et al. 2018). Los microorganismos endófitos se asocian con plantas hospederas compatibles y brindan beneficios a través de la producción de fitohormonas, la suplementación de nutrientes y la supresión del acceso de patógenos al hospedero (Singh et al. 2020). El género Bacillus es uno de los más predominantes que se encuentra en el suelo, y se han reportado diferentes especies en diversos nichos ecológicos; se sabe que los miembros de este género tienen múltiples rasgos benéficos que ayudan a las plantas directa 0 indirectamente a través de la adquisición de nutrientes, inducción del crecimiento mediante la producción de fitohormonas, protección contra patógenos y otros factores abióticos (Saxena et al. 2019). Las especies de Bacillus muestran actividad antimicrobiana de amplio espectro y se han utilizado como agentes de control biológico en la agricultura (Chen et al. 2020). Recientemente, se ha reportado que $B$. amyloliquefaciens posee actividad de biocontrol y es eficaz para controlar patógenos como: Erysiphe cichoracearum, Phytophthora parasitica var. nicotianae, Fusarium avenaceum f. sp. fabae, Streptomyces scabies, Monilinia fructigena, F. graminearum, Gaeumannomyces graminis, Setosphaeria turcica, Verticillium dahliae, Alternaria alternata, Fusarium oxysporum f.sp. lycoperisci, Botrytis cinerea y F. solani (Jiao et al. 2020). Por lo cual, el objetivo de esta investigación fue conocer la actividad antifúngica de dos aislamientos endófitos de Bacillus amyloliquefaciens contra Fusarium verticillioides bajo condiciones de laboratorio e invernadero.

\section{MATERIALES Y MÉTODOS}

\section{Aislamiento de bacterias endófitas}

El aislamiento se realizó de plantas de papa variedad Fiana de acuerdo con la metodología de Pérez et al. (2010). Las cepas se caracterizaron bioquímicamente de acuerdo con las características reportadas por Schaad et al. (2001) y se mantuvieron en refrigeración a $4{ }^{\circ} \mathrm{C}$ hasta su corroboración molecular.

\section{Aislamiento e identificación del fitopatógeno}

F. verticillioides se aisló de plantas de maíz con síntomas de marchitez y acame. El hongo se identificó por sus características morfológicas y fenotípicas en medio de cultivo PDA y Carnation Leaf Agar (CLA) (Leslie y Summerell 2006).

\section{Corroboración molecular de las cepas}

La cepa de F. verticillioides fue cultivada durante 12 días en medio Czapek-Dox (Correll et al. 1989), las bacterias en caldo LB durante $48 \mathrm{~h}$ en 
un agitador rotatorio a $26{ }^{\circ} \mathrm{C}$. La extracción del ADN genómico se realizó con la metodología de Nicholson et al. (2001). La secuenciación de las regiones parciales del gen 16S rRNA e ITS1-ITS4 se llevó a cabo por el laboratorio Macrogen. Las secuencias obtenidas se compararon con las reportadas en la base de datos del NCBI del GenBank.

\section{Capacidad antagonista in vitro}

Se estableció un ensayo en cultivo dual por triplicado (Hernández-Castillo et al. 2014). La inhibición, se calculó con la siguiente ecuación: $P I=100-[(C r * 100) / R p]$. Donde: $\mathrm{PI}=$ inhibición del crecimiento del hongo, $\mathrm{Cr}=$ crecimiento micelial del hongo y $\mathrm{Rp}=$ radio de la placa (HernándezCastillo et al. 2008).

\section{Efecto del biocontrol in planta bajo condiciones de invernadero}

El cultivo de maíz (UA-Vanta) se desarrolló en un invernadero con cubierta de polipropileno. Se utilizaron macetas de $10 \mathrm{Kg}$ de capacidad con suelo pasteurizado. Al momento de la siembra se inocularon $25 \mathrm{~mL}$ de la suspensión de $1 \times 10^{9}$ conidios $\mathrm{mL}^{-1}$ de $F$. verticillioides y simultáneamente aspersión de $1 \times 10^{8}$ ufc $\mathrm{mL}^{-1}$ de $B$. amyloliquefaciens al momento de la siembra, la segunda aplicación cuando las plantas alcanzaron $15 \mathrm{~cm}$ de altura y la última 15 días después de la segunda. Los tratamientos evaluados fueron: $(F v+B a 21) F$. verticillioides + B. amyloliquefaciens cepa 21, (Fv+Ba 53) $F$. verticillioides $+B$. amyloliquefaciens cepa 53, (Ba 21) B. amyloliquefaciens cepa 21, (Ba 53) B. amyloliquefaciens cepa 53, (Fv) Fusarium verticillioides, $(\mathrm{T})$ Testigo absoluto. El experimento se estableció en un diseño completamente al azar con 10 repeticiones por tratamiento. La incidencia de la enfermedad se evaluó como porcentaje; la severidad con una escala arbitraria de seis clases reportada por Castro et al. (2017).

\section{B. amyloliquefaciens en la promoción de crecimiento}

Cien días después de la emergencia se determinó el peso del fruto en etapa R2, diámetro de tallo, peso seco del follaje y el contenido de clorofila en las hojas expresado en unidades SPAD.

\section{Análisis estadístico}

Las diferencias en inhibición, incidencia de la enfermedad, el peso del fruto, diámetro de tallo, peso seco del follaje y el contenido de clorofila se determinaron por la prueba de medias de Tukey $(P=0.05)$. La severidad fue analizada con la prueba de KruskallWallis. Los análisis se realizaron con el programa SAS versión 9.0.

\section{RESULTADOS Y DISCUSIÓN}

\section{Aislamiento e identificación de las cepas}

Se aislaron 28 cepas de bacterias endófitas, de las cuales Bacillus amyloliquefaciens cepa 21 y B. amyloliquefaciens cepa 53 presentaron el más alto porcentaje de inhibición; las cuales se identificaron con tinción de Gram positiva, presencia de flagelos perítricos, prueba de RYU y oxidasa negativa, resultado positivo en catalasa, oxidación, fermentación y motilidad. La espora se encontró al centro del cuerpo bacteriano. Crecieron a temperaturas de $45{ }^{\circ} \mathrm{C}-65$ ${ }^{\circ} \mathrm{C}$, en $\mathrm{pH} 5.7$ y en concentraciones de $\mathrm{NaCl}$ a $7 \%$, pero no en $5 \%$ y $3 \%$. Tuvieron crecimiento anaeróbico en glucosa. Produjeron ácido a partir de arabinosa y negativo en manitol. Fueron positivas en la reacción Voges-Proskauer, hidrólisis de almidón y citrato. La corroboración molecular se basó en el análisis de la secuencia del gen $16 \mathrm{~S}$ rRNA, mostrando $99 \%$ de homología con las secuencias contenidas en la base de datos del GenBank con clave de acceso KU570451.1 y KX665550.1, respectivamente.

F. verticillioides se aisló de tallos de maíz infectados y se identificó en base a la formación de microconidios y macroconidios (Leslie y Summerell 2006, Sameer et al. 2018); la corroboración molecular arrojó $99.60 \%$ de identidad con las secuencias de F. verticillioides cepa QN0629 contenida en el Genbank con clave de acceso MN121060. El hongo $F$. verticillioides se identificó como el agente causal de pudrición de tallo y acame en el cultivo del maíz lo que coincide con reportes realizados por Figueroa- 
López et al. (2016).

\section{Capacidad antagonista in vitro}

Los resultados de la actividad antifúngica se reportan visualmente en la Figura 1, representa la experimentación con clara indicación de la zona de inhibición que fue medida en centímetros y comparados con el testigo. La inhibición de crecimiento radial del patógeno causado por las cepas antagonistas osciló de 59 a 62\%. La máxima inhibición de crecimiento radial se detectó con la cepa B. amyloliquefaciens cepa 53 contra F. verticillioides con $62 \%$ de inhibición, seguida de la cepa 21 (59\%). Otros aspectos de la interacción entre los antagonistas y el fitopatógeno durante el ensayo de cultivo dual fue notorio que a partir del sexto día se detuvo el crecimiento del hongo, indicando una alta competencia por el espacio y los nutrientes (Figura 2).

La capacidad de inhibición de Bacillus se atribuye a la producción de compuestos antimicrobianos, como policétidos; síntesis de enzimas líticas, producción de hormonas, de compuestos orgánicos volátiles, competencia por espacio y nutrientes e inducción de resistencia sistémica (Pedraza et al. 2020). Bacillus amyloliquefaciens puede actuar contra F. verticillioides, F. oxysporum, Sclerotinia sclerotiorum, F. proliferatum, Colletotrichum gloeosporioides, F. equiseti y F. semitectum (Xu et al. 2021). Otros géneros como Bacillus, Lysinibacillus, Brevibacillus, Geobacillus, Paenibacillus y Terribacillus también son antagonistas de $F$. verticillioides (Figueroa-López et al. 2016).

\section{Efecto de biocontrol in planta}

$B$. amyloliquefaciens redujo significativamente la incidencia y severidad del fitopatógeno $(P<$ 0.0001 ). La cepa 53 presentó $20 \%$ de incidencia, mientras que la cepa 21 presenta $80 \%$ en comparación con las plantas inoculadas solo con el patógeno (100\%). La severidad fue de 2.5 en los tratamientos de la cepa 53 y de 14.38 en la cepa 21 con relación al patógeno (40.83). Al respecto Martínez-Álvarez et al. (2016) reportan una formulación a base de Bacillus capaz de controlar F. verticillioides en invernadero. El recubrimiento de semillas de tomate con bacte- rias endofíticas redujo la incidencia y severidad de $F$. oxysporum en campo y estimularon el crecimiento de las plantas (Koohakan et al. 2020). En adición a lo anterior, se puede mencionar que las cepas de los antagonistas endófitos estudiados son promisorios para reducir la severidad de la enfermedad contra $F$. verticillioides.

\section{B. amyloliquefaciens en la promoción de crecimiento}

El análisis en la actividad de las cepas de $B$. amyloliquefaciens contra $F$. verticillioides incrementó significativamente el contenido de clorofila en comparación con el testigo (Tabla 1). La clorofila es un pigmento fotosintético importante para la planta, que determina en gran medida la capacidad fotosintética y, por lo tanto, el crecimiento de la planta (Li et al. 2018). La inoculación de plantas con $B$. cereus, B. subtilis y $B$. amyloliquefaciens influyó positivamente en el desempeño del mecanismo fotosintético en plantas de pimiento para aumentar la fluorescencia de la clorofila y los parámetros de intercambio de gases (Samaniego et al. 2016). La aplicación de $B$. thuringiensis y quitosano mejoró el crecimiento de plantas de pimiento y aumentó significativamente el contenido de clorofila, el parámetro de fluorescencia de la clorofila y el rendimiento de frutos (ALKahtani et al. 2020). La degradación de la clorofila se reduce en hojas de arroz por el efecto de Xanthomonas oryzae pv. oryzae durante la infección (Atta et al. 2018), lo que demuestra que las plantas enfermas por algún fitopatógeno tienen menos clorofila que las sanas. Por su parte, Cunha et al. (2015) mencionan que el período de más necesidad de nitrógeno es establecido en función del SPAD, comprobado al realizar la fertilización nitrogenada. Es importante mencionar que el experimento se mantuvo libre de fertilización química y los valores más altos del contenido de clorofila es por el efecto de la inoculación de las bacterias endófitas.

Dentro de las bondades del genero Bacillus se encuentra la promoción del crecimiento vegetal; por lo que las plantas de maíz inoculadas con las cepas endófitas de $B$. amyloliquefaciens en ausencia del fitopatógeno se observó un incremento del peso fresco 


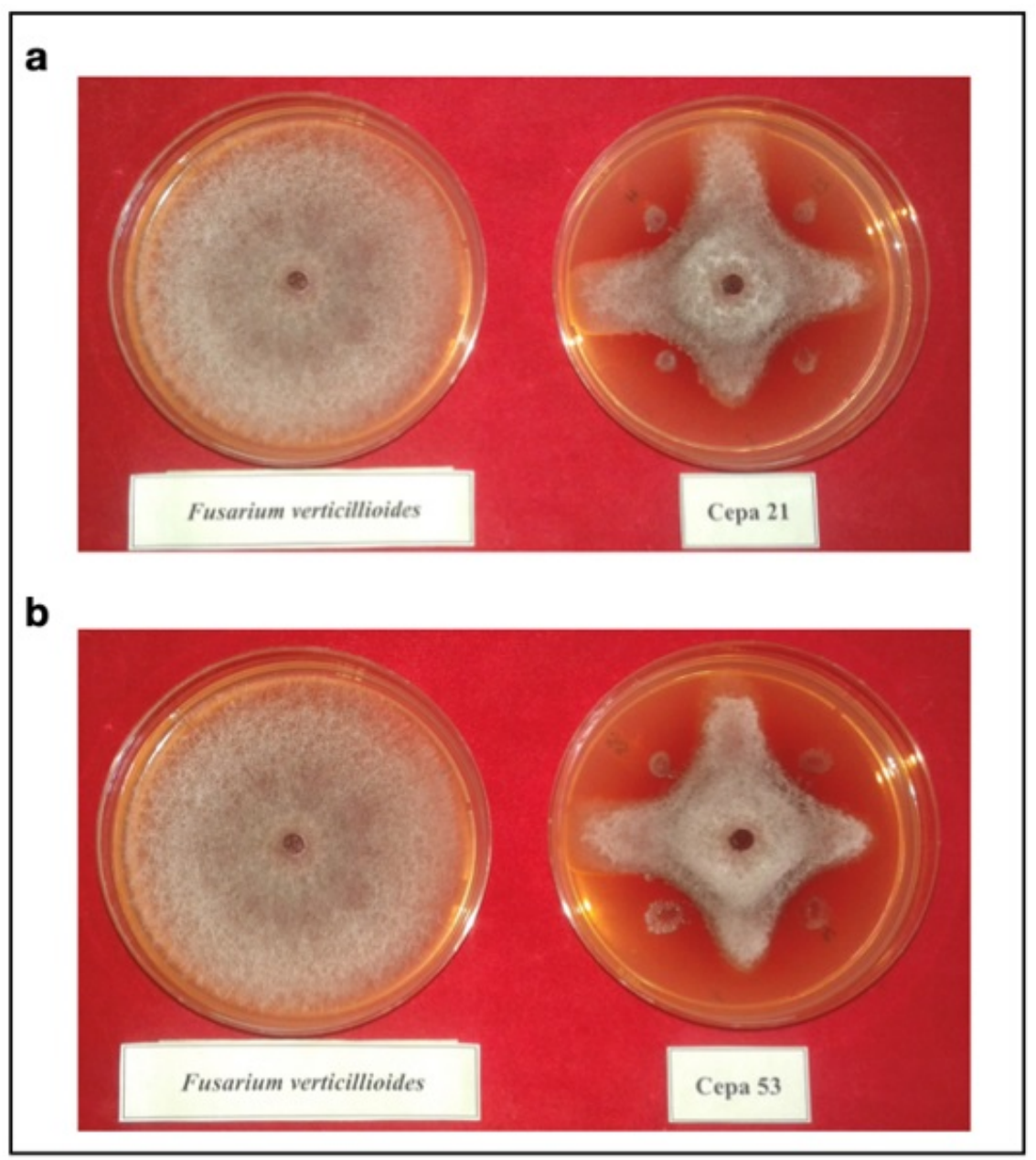

Figura 1. Antagonismo in vitro de Bacillus amyloliquefaciens cepa 21 (a) y B. amyloliquefaciens cepa 53 (b) sobre el crecimiento micelial de F. verticillioides en cultivos duales, evaluado a los 11 días de incubación.

del fruto, diámetro de tallo y peso seco de biomasa aérea en comparación con el testigo (Tabla 1). La mejora de estas variables fue notoria por la cepa 53 al sobreponerse sobre el testigo y el resto de los tratamientos. Bacillus produce 1-aminociclopropano1-carboxilato (ACC) desaminasa que confiere la inducción de tolerancia al estrés por sequía y promoción del crecimiento vegetal en Solanum lycopersicum (Gowtham et al. 2020). La altura y el peso fresco de biomasa de plántulas de $S$. lycopersicum se pueden incrementar con la aplicación de filtrados libres de células de B. subtilis (Cabello et al. 2019). El género Bacillus exhibe propiedades bioquímicas como la solubilización de fosfatos, producción de ácido indolacético y actividad ACC deaminasa, relacionadas con la promoción del crecimiento vegetal (Chávez-Ambriz et al. 2016). Por lo que es importante mencionar que el experimento se mantuvo libre de la aplicación de agroquímicos (fertilizantes y plaguicidas). Por lo que la cepa 53 sobresalió por su efecto estimulador del crecimiento y desarrollo de las plantas de maíz en invernadero.

Se aisló e identificó a $F$. verticillioides como agente causal de pudrición y acame del cultivo de maíz. La cepa 53 de Bacillus amyloliquefaciens exhibió una alta actividad antagonista contra F. verticillioides en cultivos duales. La incidencia y severidad de $F$. verticillioides se redujo en las plantas inoculadas 


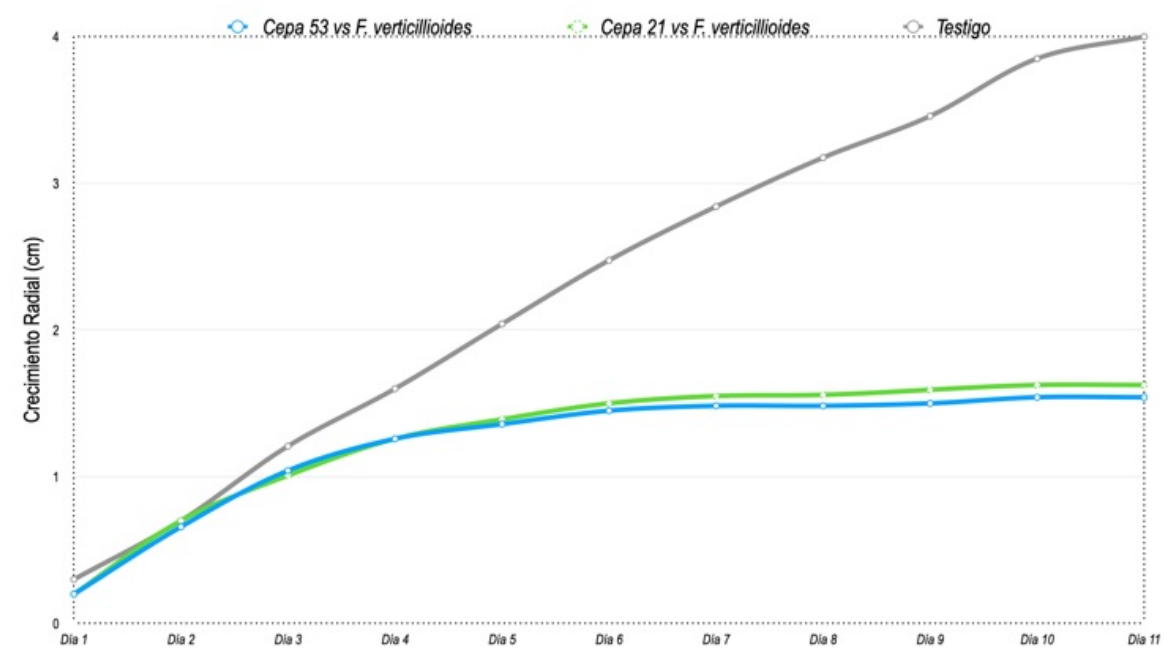

Figura 2. Dinámica de crecimiento de Fusarium verticillioides.

Tabla 1. Inducción de crecimiento de los agentes de control biológico en el ensayo de invernadero.

\begin{tabular}{lcccc}
\hline Tratamiento & $\begin{array}{c}\text { Clorofila } \\
(\mathrm{spad})\end{array}$ & $\begin{array}{c}\text { Peso fresco de fruto } \\
(\mathrm{gr})\end{array}$ & $\begin{array}{c}\text { Diametro de tallo } \\
(\mathrm{cm})\end{array}$ & $\begin{array}{c}\text { Peso seco de biomasa } \\
(\mathrm{gr})\end{array}$ \\
\hline $\mathrm{Fv}+\mathrm{Ba} 21$ & $37.5^{a b}$ & $7.3^{a}$ & $5.2^{a}$ & $7.6^{a}$ \\
$\mathrm{Fv}+\mathrm{Ba} 53$ & $37.6^{a b}$ & $9.2^{a}$ & $5.0^{a}$ & $9.5^{a}$ \\
$\mathrm{Ba} \mathrm{21}$ & $37.9^{a b}$ & $8.9^{a}$ & $5.3^{a}$ & $9.7^{a}$ \\
$\mathrm{Ba} \mathrm{53}$ & $40.3^{a}$ & $10.5^{a}$ & $5.4^{a}$ & $10.8^{a}$ \\
$\mathrm{FV}$ & $28.1^{a b}$ & $5.7^{a}$ & $4.8^{a}$ & $5.8^{a}$ \\
$\mathrm{~T}$ & $25.4^{b}$ & $6.9^{a}$ & $4.1^{a}$ & $7.0^{a}$ \\
\hline \multicolumn{2}{l}{ Medias con la misma letra no son significativamente diferentes de acuerdo a la prueba de Tukey, } \\
$\mathrm{P}=0.05$.
\end{tabular}

con las cepas de B. amyloliquefaciens. Las cepas endófitas tuvieron efecto positivo en la inducción de crecimiento del cultivo de maíz bajo condiciones de invernadero.

\section{AGRADECIMIENTOS}

Al Consejo Nacional de Ciencia y Tecnología (CONACYT) por la beca otorgada a Epifanio Castro del Ángel para realizar el Doctorado en Parasitología Agrícola en la Universidad Autónoma Agraria Antonio Narro, Saltillo, México.

\section{LITERATURA CITADA}

Anguiano Cabello JC, Flores Olivas A, Olalde Portugal V, Arredondo Valdés R, Laredo Alcalá El (2019) Evaluation of Bacillus subtilis as promoters of plant growth. Revista Bio Ciencias 6:e418. DOI: 10.15741/revbio.06.e418.

Ahangarkani F, Rouhi S, Azizi IG (2014) A review on incidence and toxicity of fumonisins. Toxin Reviews 33 : 95-100.

ALKahtani MDF, Attia KA, Hafez YM, Khan N, Eid AM, Ali MAM, Abdelaal KAA (2020) Chlorophyll fluorescence parameters and antioxidant defense system can display salt tolerance of salt acclimated sweet pepper plants treated with chitosan and plant growth promoting rhizobacteria. Agronomy 10(8): 1180. DOI: 10.3390/agronomy 10081180 
Atta BM, Saleem M, Ali H, Arshad HMI, Ahmed M (2018) Chlorophyll as a biomarker for early disease diagnosis. Laser Physics 28(6): 065607. DOI: 10.1088/1555-6611/aab94f

Caglayan MO, Üstündağ Z (2020) Detection of zearalenone in an aptamer assay using attenuated internal reflection ellipsometry and it's cereal sample applications. Food Chemistry Toxicology 136: 11108. DOI: 10.1016/j.fct.2019.111081

Castro del AE, Hernández CFD, Ochoa FYM, Gallegos MG, Castillo RF, Tucuch CFM (2017) Endophytic bacteria controlling Fusarium oxysporum and Rhizoctonia solani in Solanum tuberosum. European Journal of Physical and Agricultural Sciences 5: 29-39.

Chen K, Tian Z, He H, Long C, Jiang F (2020). Bacillus species as potential biocontrol agents against citrus diseases. Biological Control 151: 104419. DOI: 10.1016/j.biocontrol.2020.104419

Chávez-Ambriz LIA, Hernández-Morales A, Cabrera-Luna JA, Luna-Martínez L, Pacheco-Aguilar JR (2016) Aislados de Bacillus provenientes de la rizósfera de cactus incrementan la germinación y la floración en Mammillaria spp. (Cactaceae). Revista Argentina de Microbiología 48: 333-341.

Correll JC, Klittich CJR, Leslie JF (1989) Heterokaryon self-incompatibility in Gibberella fujikuroi (Fusarium moniliforme). Mycological Research 93: 21-27.

Cunha AR, Katz L, Sousa AP, Martinez URA (2015) Indice SPAD en el crecimiento y desarrollo de plantas de lisianthus en función de diferentes dosis de nitrógeno en ambiente protegido. Idesia (Arica) 33: 97-105.

Einloft TC, Hartke S, de Oliveira PB, Silveira SP, Gomes DR (2021) Selection of rhizobacteria for biocontrol of Fusarium verticillioides on non-rhizospheric soil and maize seedlings roots. European Journal of Plant Pathology 160: 503-518.

Figueroa-López AM, Cordero-Ramírez JD, Martínez-Álvarez JC, López-Meyer M, Lizárraga-Sánchez GJ, FélixGastélum R, Castro-Martínez C, Maldonado-Mendoza IE (2016) Rhizospheric bacteria of maize with potential for biocontrol of Fusarium verticillioides. Springer Plus 5: 1-12. DOI: 10.1186/s40064-016-1780-x

Gowtham HG, Singh SB, Murali M, Shilpa N, Prasad M, Aiyaz M, Amruthesh KN, Niranjana SR (2020) Induction of drought tolerance in tomato upon the application of ACC deaminase producing plant growth promoting rhizobacterium Bacillus subtilis Rhizo SF 48. Microbiological Research 234: 126422.

Hernández-Castillo FD, Lira-Saldivar RH, Cruz-Chávez L, Gallegos-Morales G, Galindo-Cepeda ME, PadrónCorral E, Hernández-Suárez M (2008) Antifungal potential of Bacillus spp. strains and Larrea tridentata extract against Rhizoctonia solani on potato (Solanum tuberosum L.) crop. Phyton International Journal of Experimental Botany 77: 214-252.

Hernández-Castillo FD, Lira-Saldivar RH, Gallegos-Morales G, Hernández-Suárez M, Solís-Gaona S (2014) Biocontrol of pepper wilt with three Bacillus species and its effect on growth and yield. Phyton International Journal of Experimental Botany 83: 49-55.

Jiao R, Munir S, He P, Yang H, Wu Y, Wang J, He P, Cai Y, Wang G, He Y (2020) Biocontrol potential of the endophytic Bacillus amyloliquefaciens YN201732 against tobacco powdery mildew and its growth promotion. Biological Control 143: 104160. DOI: 10.1016/j.biocontrol.2019.104160

Kamle M, Mahato DK, Devi S, Lee KE, Kang SG, Kumar P (2019) Fumonisins: impact on agriculture, food, and human health and their management strategies. Toxins 11: 1-23. DOI: 10.3390/toxins11060328

Koohakan P, Prasom P, Sikhao P (2020) Application of seed coating with endophytic bacteria for Fusarium wilt disease reduction and growth promotion in tomato. International Journal of Agricultural Technology 16: 55-62. 
Kuki MC, Pinto RJB, Bertagna FAB, Tessmann DJ, Teixeira do Amaral A, Scapim CA, Holland JB (2020 Association mapping and genomic prediction for ear rot disease caused by Fusarium verticillioides in a tropical maize germoplasm. Crop Science 60: 2867-2881.

Leslie JF, Summerell BA (2006) The Fusarium Laboratory Manual. Blackwell Publishing. USA. 388p.

Li Y, He N, Hou J, Xu L, Liu C, Zhang J, Wang Q, Zhang X, Wu X (2018) Factors influencing leaf chlorophyll content in natural forests at the biome scale. Frontiers in Ecology and Evolution 6: 1-10. DOI: 10.3389/fevo.2018.00064

Liu X, Fan L, Yin S, Chen H, Hu H (2019) Molecular mechanisms of fumonisin B1-induced toxicities and its applications in the mechanism-based interventions. Toxicon 167: 1-5. DOI: 10.1016/j.toxicon.2019.06.009

Martínez-Álvarez JC, Castro-Martínez C, Sánchez-Peña P, Gutiérrez-Dorado R, Maldonado-Mendoza IE (2016) Development of a powder formulation based on Bacillus cereus sensu lato strain B25 spores for biological control of Fusarium verticillioides in maize plants. World Journal of Microbiology and Biotechnology 32: 75. DOI: $10.1007 / \mathrm{s} 11274-015-2000-5$

Nasir HA, Abbasi S, Sharifi, R, Jamali S (2018) The effect of biocontrol agents consortia against Rhizoctonia root rot of common bean Phaseolus vulgaris. Journal of Crop Protection 7: 73-85.

Nicholson TP, Rudd BAM, Dawson M, Lazarus CM, Simpson TJ, Cox RX (2001) Design and utility of oligonucleotide gene probes for fungal polyketide synthases. Chemistry and Biology 8: 157-178.

Pedraza LA, López CA, Uribe-Vélez D (2020) Mecanismos de acción de Bacillus spp. (Bacillaceae) contra microorganismos fitopatógenos durante su interacción con plantas. Acta Biológica Colombiana 25: 112125.

Pérez A, Rojas J, Fuentes J (2010) Diversidad de bacterias endófitas asociadas a raíces del pasto colosuana (Bothriochloa pertusa) en tres localidades del departamento de Sucre, Colombia. Acta Biológica Colombiana 15: 219-228.

Samaniego-Gámez BY, Garruña R, Tun-Suárez JM, Kantun-Can J, Reyes-Ramírez, Cervantes-Díaz L (2016) Bacillus spp. inoculation improves photosystem II efficiency and enhances photosynthesis in pepper plants. Chilean Journal of Agricultural Research 76: 409-416.

Sameer K, Sobanbabu G, Sabarinathan KG, Parthiban VK, Ramamoorthy V (2018) Evaluation of phyllosphere antagonistic bacteria on the management of Fusarium ear rot of maize caused by Fusarium verticillioides. Journal of Biological Control 32: 257-263.

Saxena KA, Murugan K, Chakdar H, Anuroopa N, Bagyaraj D (2019) Bacillus species in soil as a natural resource for plant health and nutrition. Journal of Applied Microbiology 128: 1583-1594.

Singh M, Srivastava M, Kumar A, Singh A K, Pandey KD (2020) 4- Endophytic bacteria in plant disease management. In: Ajay Kumar, Vipin Kumar Singh, (eds) Woodhead Publishing Series in Food Science, Technology and Nutrition, Microbial Endophytes, Woodhead Publishing, pp: 61-89. DOI: 10.1016/b978-0-12-8187340.00004-8

Schaad NW, Jones JB, Chun W (2001) Laboratory guide for identification of plant pathogenic bacteria. APS Press. Minessota. 373p.

Wang Y, Zhou Z, Gao J, Wu Y, Xia Z, Zhang H, Wu J (2016) The mechanisms of maize resistance to Fusarium verticillioides by comprehensive analysis of RNA-seq data. Frontiers in Plant Science 7: 1654. DOI: 10.3389/fpls.2016.01654 
Xu S, Wang Y, Hu J, Chen X, Qiu Y, Shi J, Wang G, Xu J (2021) Isolation and characterization of Bacillus amyloliquefaciens MQ01, a bifunctional biocontrol bacterium with antagonistic activity against Fusarium graminearum and biodegradation capacity of zearalenone. Food Control 130: 108259. DOI: 10.1016/j. foodcont.2021.108259. 